\title{
Frank Lehman, Hollywood Harmony: Musical Wonder and the Sound of Cinema
}

New York: Oxford University Press, 2018 [xvii, 292 pp. ISBN: $9780190606404 \$ 39.95$ (trade paper)]. The Oxford Music/Media Series.

\author{
ERIK HEINE \\ Oklahoma City University \\ eheine@okcu.edu
}

I

$\mathrm{n}$ the past twenty years, scholarship of film music, both musicological and analytical, has exploded, due in part to my generation's interest in film music as well as the fact that film music is "serious music." It is now commonplace to find articles concerning aspects of film music in top-tier journals, as well as monographs and anthologies. Frank Lehman's book Hollywood Harmony is another addition to the growing field of film music studies, and investigates triadic film music through the interlocutor of the broader discipline of music theory.

The main arguments in Hollywood Harmony - that "pitch design is enlisted in film for its expressive and associative powers over its unifying structural capabilities" and "[p]antriadic harmony is a potent style topic, used throughout film history to represent and sometimes elicit the affect of wonderment"are clearly elucidated in the Introduction (pp. 8, 10). Lehman then presents the book's organization, moving from style to theory and then to close readings of cues, grouping the six chapters into three units: practices and tools, interpretive questions, and aesthetics and interactions. It is in the final chapter, in which Lehman proposes a new model of "triadic tonal space," derived from Dmitri Tymoczko's musicalgeometrical model, that he accounts for diatonicity, centricity, and functionality.

The opening chapters focus on musical style and analytical tools. In Chapter 1, "Tonal Practices," Lehman illustrates the various tools at the disposal of most music theorists, applying them to cues from across decades and styles. Lehman also addresses referentiality, stating that, while composers draw on earlier styles to make connections to concert music (Jerry Goldsmith drawing from the octatonic scale and polyrhythms in a fashion similar to Igor Stravinsky's Symphony in Three Movements), film music also has the ability to be non-referential. The focus of Chapter 2, "Expression and Transformation," is pantriadicism. Lehman begins with historical models from Richard Wagner, and moves into scale collections such as whole-tone, octatonic, and hexatonic, all the while foregrounding transpositions in his musical examples. Lehman also provides the aesthetic results of the use of pantriadicism, such as intensification, fantasy, atypical psychology, and sublimity. Lehman briefly introduces NeoRiemannian Theory (NRT) in this chapter, which serves as a segue into Chapter 3, "Neo-Riemannian Theory at the Movies."

Chapter 3 begins the theory-focused portion of the book, and is divided into two halves, with the first a textbook-like introduction to Neo-Riemannian Theory. Following this primer, Lehman introduces annotated examples and diagrams to aid the reader in understanding how to apply Neo-Riemannian Theory to film music and how to interpret its associative connections. "Analyzing Chromaticism in Film," Chapter 4, is where analysis becomes foregrounded. Lehman invites his audience to participate in the analysis by encouraging the reader to play through the musical examples on the piano in order to have a sense of creating the music, rather than just listening, emphasizing activity rather than passivity. Much of the chapter is highly technical, using 
NRT transformations, but Lehman's prose deftly leads the reader through the examples, and each example is meticulously annotated, often with the film's action noted above the score. A reader could play the example, note the action, and be able to approximate the film without having to find a DVD or even an online clip. The examples in the chapter draw on multiple decades, genres, and composers, strengthening Lehman's analysis through their breadth and range.

The focus of Chapter 5, "Pantriadic Wonder," is on the concepts of frisson and awe as they relate to broader issues of amazement and wonder and their ability to be used within a tonal framework. Lehman draws on work from music theorists, as well as psychologist Marilyn Boltz and philosopher Philip Fisher, as issues of musical expectation and temporality are significant to creating wonder, awe, and frisson in audiences. Lehman presents these wondrous ideas, specifically awe, through an example from The Matrix. Lehman takes this example, originally presented by David Huron, and applies his own transformational labels to build an explanation of the music within a pantriadic context. ${ }^{1}$ Much of the remainder of the chapter centers on close readings of cues from the Lord of the Rings trilogy, with examples drawn from all three films. Beyond these close musical readings, Lehman provides figures that show exactly how the desired reactions, awe and frisson, respectively, work within musical transformational networks, expectancy, and temporality. This chapter is a significant contribution to the field, because these concepts have rarely, if ever, been codified and presented in such a precise manner; and could easily serve as the basis for an additional monograph that investigates these concepts across films within a specific genre, or across the output of a single composer.

Chapter 6, "Harmonious Interactions," is the book's final chapter, where Lehman posits a theory of triadic chromaticism and harmonic syntax. He draws on the musical geometry work of Tymoczko to create a three-dimensional representation of triadic tonality space, where common practice tonality and pantriadic chromaticism are on opposite corners, as they should be. Lehman provides examples from composers whose tendencies are placed on specific corners of the diagram, allowing compositional style to be represented by the markers, followed by three short "intraphrasal chromaticism" examples, all three

1 See David Huron, Sweet Anticipation: Music and the Psychology of Expectation (Cambridge, MA: MIT Press, 2006), 289. illustrating different methodologies of beginning and ending within the same tonality, but differing in how the process is achieved. Triadic tonal space is used to support Lehman's claim for tonal dualism, and he draws on examples that illustrate different forms of tonal interaction, all leading to the "beatific sublime," where the music is, "somewhat more apt to play upon the diatonic/chromatic polarity than music for less theologically motivated flights of fancy" (p. 223). The chapter culminates with 'The Vision', a cue from Alfred Newman's score for the 1943 religious drama The Song of Bernadette. The cue, over four minutes long, ties together several analytical threads that have been presented throughout the book, and, much like the organization of a film, the climactic moment happens right before the final credits. To provide a brief repose before the book's "end credits," Lehman offers a short "Afterword," offering an example from the end of the final installment of the Lord of the Rings trilogy, providing the appropriate bookend to the monograph's opening, which used an example from the Universal Pictures Logo. He acknowledges that the book investigates only a small portion of the genre, as well as a number of possibilities for further research for those willing to build upon this foundation.

The various types of analyses in the book draw on music theory methodologies such as Schenkerian Analysis, cadential styles and gestures, scale type, and, most importantly, Neo-Riemannian Theory. Lehman gives NRT an entire chapter to be explained, both for the musical layperson and for the less experienced music theorist. In addition to the three standard operations, Lehman introduces additional operations to demonstrate more distant relationships for chord roots not separated by thirds. The time spent on creating a lexicon for NRT is substantial, but it creates an ease of understanding for the musically annotated examples throughout the book in a harmonically non-functional environment.

The book contains dozens of musical examples, all transcribed and reduced by Lehman, and accompanying audio and film examples are available through the website provided in the book. These examples are essential due to the variety of genres and composers on which Lehman draws. In this way, the accompanying website is invaluable, as readers can hear the musical examples in isolation, and also see how they are used within the film's larger structure. The largest liability in Lehman's book is that its scope limits the types of examples from which it can draw: mainly films that evoke wonder, amazement, and a sense of the fantastic. These films 
will not necessary speak to all readers, and some might look at the relative lack of examples from "important" film composers covered in the booksuch as Bernard Herrmann and John Williams-or a lack of examples from dramatic and "serious" films as evidence that the book fails to address more intricate and polyphonic music, or perhaps that film music is not "serious music." However, Lehman's analytical depth is incredible, and his prose is elegant and logical. His transcriptions are outstanding, and his ability to draw on examples from the 1930s through 2016 demonstrates that pantriadicism has been in use throughout Hollywood's harmonic history. Hollywood Harmony is indispensable to those interested in film music analysis, particularly those who teach at the college level, and will serve as the basis for a great deal of scholarship in the next twenty years.
Erik Heine is Professor of Music at Oklahoma City University, where he has taught since 2005. Additionally, he is the Assistant Director of the Honors Program. His primary research area is in film music, and he has published articles, book chapters, and a monograph on various aspects of film music and music theory. He holds a PhD in Music Theory from the University of Texas at Austin. 